\section{Commentary: Postpericardiotomy syndrome remains an enigma}

\author{
Kevin L. Greason, MD
}

Readers of the Journal will find interest in this article on postpericardiotomy syndrome (PPS) after surgical aortic valve replacement from the CAREFIB group of investigators. ${ }^{1}$ The current study continues the CAREFIB group's interest in the syndrome. The article is well written and provides insight into PPS.

The authors report on 671 patients operated with isolated surgical aortic valve replacement. PPS was defined by at least 2 of the following: (1) fever without alternative cause, (2) pericarditic or pleuritic chest pain, (3) pericardial or pleural rubs, (4) pericardial effusion, and/or (5) pleural effusion with elevated C-reactive protein. PPS was categorized as moderate when no invasive intervention occurred and severe when invasive intervention occurred (eg, pericardial or pleural drainage).

The median duration of follow-up was 9.0 (6.1-12.0) years. PPS developed in $75(11.1 \%)$ patients and was classified moderate in $51(7.6 \%)$ and severe in $24(3.5 \%)$ of patients. The median time to diagnosis was 16 (11-36) days. Interventions in the severe PPS group included evacuation of a pericardial effusion in 15 patients and evacuation of a pleural effusion in 11 patients.

The authors reported that patients with severe PPS had increased all-cause mortality (adjusted analysis: hazard ratio 2.01 but rather wide $95 \%$ confidence interval of 1.03-3.91; $P=.040)$ with a median time from diagnosis to death of 460 (83-1300) days. Correlation may not imply causation. The listed causes of death are variable, and the numbers do not easily add up to the reported total of 7. PPS was listed as cause of death in only 1 patient. Pneumonia, atherosclerotic heart disease, and aortic valve stenosis were also listed as causes of death in apparently several patients.

In a previous publication on PPS after open-heart operations, the CAREFIB group excluded from analyses patients

\footnotetext{
From the Department of Cardiovascular Surgery, Mayo Clinic, Rochester, Minn Disclosures: Author has nothing to disclose with regard to commercial support.

Received for publication Jan 25, 2020; accepted for publication Jan 26, 2020; available ahead of print Feb 7, 2020.

Address for reprints: Kevin L. Greason, MD, Department of Cardiovascular Surgery,

Mayo Clinic, 200 First St, Rochester, MN 55905 (E-mail: greason.kevin@mayo. edu).

J Thorac Cardiovasc Surg 2020;160:1457 0022-5223/\$36.00

Copyright (c) 2020 Published by Elsevier Inc. on behalf of The American Association for Thoracic Surgery

https://doi.org/10.1016/j.jtcvs.2020.01.072
}

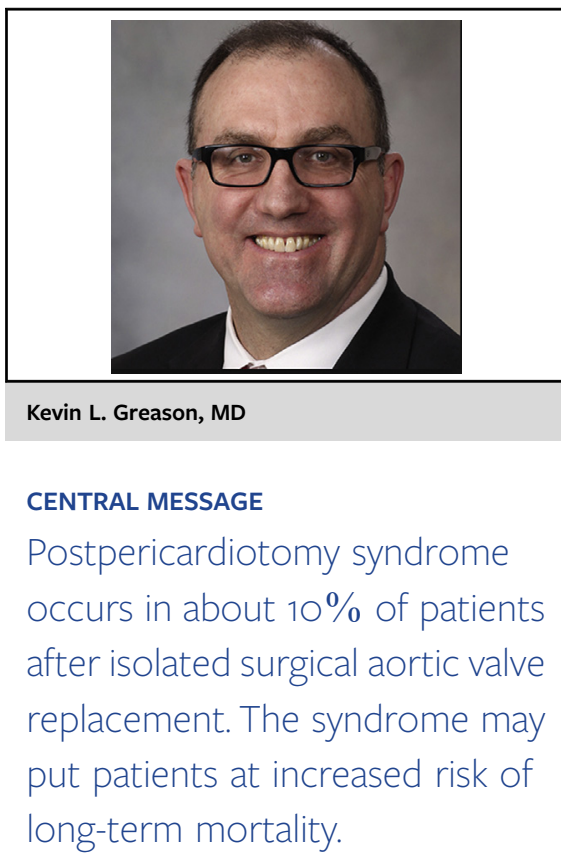

who died within 30 days after surgery. ${ }^{2}$ The authors state this was done to minimize the false-negative diagnoses. Such was not done in the current analysis. Examination of Figure 4 demonstrates an early drop in the survival curves suggestive of increased operative mortality in the severe and no-PPS groups. Should operative mortalities have been excluded?

The authors reported that the first-line medication use was glucocorticoids in $64 \%$ of the patients with PPS, whereas only $20 \%$ received colchicine. This treatment paradigm is counter to current guidelines, which recommend colchicine as the first-line treatment. The authors make note of this in the discussion section of the manuscript. It is unclear the impact of misdirected first-line medication use with glucocorticoids on the mortality endpoint.

The study raises several important questions in need for further study. How to differentiate postoperative pneumonia from PPS? Is there a sex-age interaction? Is there an important distinction between moderate and severe PPS? And, most importantly, how do the adverse events of PPS influence mortality? The authors recognize the issues. Further study is warranted.

\section{References}

1. Lehto J, Gunn J, Bjorn R, Malmberg M, Juhani Airaksinen KE, et al. Adverse events and survival with postpericardiotomy syndrome after surgical aortic valve replacement. J Thorac Cardiovasc Surg. 2020;160:1446-56.

2. Lehto J, Kiviniemi T, Gunn J, Airaksinen J, Rautava P, Kyto V. Occurrence of postpericardiotomy syndrome: association with operation type and postoperative mortality after open-heart operations. J Am Heart Assoc. 2018;7:e010269. 